\title{
Yellow nail syndrome
}

\section{Sudip Nanda MD, Fabio Dorville MD}

Previously published at www.cmaj.ca

$\mathrm{A}$ 58-year-old woman presented with yellowish discoloration of the fingernails and toenails (Figures 1A and $1 \mathrm{~B})$, intermittent cough and edema of both ankles (Figure 1C). The discoloration of the nails had been present for 7 years and had not responded to multiple antifungal treatments. The patient had experienced several episodes of nonproductive cough during the previous few years. Each episode had lasted 2-3 months before subsiding spontaneously. The patient had also experienced difficulty getting her shoes on for the previous 3 months.

Results of hematologic and biochemical tests were normal except for a reduced level of serum albumin (32 [normal 35-55] g/L). A computed tomography scan of the chest showed bilateral pleural effusions (Figure 1D). Thoracocentesis revealed an exudative pleural fluid with lymphocytic predominance. Echocardiography, doppler ultrasonography of the lower limbs and a computed tomography scan of the abdomen were normal. A nail biopsy was negative for fungal growth.

Yellow nail syndrome usually presents between the fourth and sixth decades of life and is thought to be an acquired condition. It may be idiopathic, secondary to another condition (e.g., connective tissue disease, a thyroid disorder or malignancy) or an adverse effect of drugs (e.g., penicillamine, bucillamine or gold sodium thiomalate). The pathogenesis is poorly understood. Structural or functional lymphatic abnormalities and increased vascular permeability to albumin are proposed pathologic mechanisms ${ }^{1}$.

Diagnosis is based on the presence of yellowish discoloration of the nails and lymphedema or respiratory manifestations. Respiratory findings may include effusions, bronchial hyper-responsiveness, bronchiectasis, bronchitis and sinusitis. The classic triad of yellow nails, lymphedema and pleural effu-

From the Department of Internal Medicine, St. Luke's Hospital, Bethlehem, USA

Cite as CMAJ 2009. DOI:10.1503/cmaj.080255 sion is simultaneously present in only a quarter of patients with the syndrome. Discoloration of the nails is the most consistent finding. A third of patients have pleural effusions.

The long-term outcome for yellow nail syndrome is variable and related to the type and severity of associated conditions. The syndrome is associated with reduced survival compared with the general population, and remissions and relapses can occur. Treatment is symptomatic. ${ }^{2}$ In our patient, reasonable control of the edema has been achieved with the use of pneumatic stockings and diuretics. A trial of vitamin E therapy was unsuccessful for the discoloured nails.

Acknowledgement: We thank Anne Kemp for the images.

\section{REFERENCES}

1. D'Alessandro A, Muzi G, Monaco A, et al. Yellow nail syndrome: does protein leakage play a role? Eur Respir J 2001;17:149-52.

2. Maldonado F, Tazelaar HD, Wang CW, et al. Yellow nail syndrome: analysis of 41 consecutive patients. Chest 2008;134:375-81. 